\section{Keeping kidneys}

An unusual explanation for the rejection of kidney transplants emerges from a study by Duska Dragun et al. in the 10 February New England Journal of Medicine (352, 558-569). The culprit is antibodies directed against the angiotensin II type 1 ( $\left.\mathrm{AT}_{1}\right)$ receptor, which, among other tasks, helps coordinate blood pressure.

Antibody-mediated mechanisms are thought to underlie about a third of acute rejection episodes. Many of these episodes can be traced to anti๑ bodies against donor HLA antigens; but episodes also are known to occur because of antibodies against other targets.

To identify such targets, the researchers studied 33 patients with symptoms of severe antibody-mediated rejection. Of these patients, 16 had severe high blood pressure - and they also had antibodies against $\mathrm{AT}_{1}$. In cell culture, antibodies against $\mathrm{AT}_{1}$ activated the receptor, and in rats, infusion of antibodies elicited symptoms of acute rejection.

The investigators reasoned that measures to counteract the antibodies — such as drugs that block the $\mathrm{AT}_{1}$ receptor-would counteract rejection in their patients. Seven individuals received such therapies, faring better than untreated individuals — although subjects were not randomly selected. Exactly how $\mathrm{AT}_{1}$ autoantibodies emerge remains unclear.

\section{Smoking out Alzheimer disease}

Studies have emerged over the last several years hinting that nicotine might counteract Alzheimer disease, providing unexpected hope to smokers. That might be overly optimistic, according to a study in mice in the 10 February Proceedings of the National Academy of Sciences (doi: 10.1073/pnas.0408500102). Salvatore Oddo et al. show that smoking seems to exacerbate one molecular aspect of Alzheimer disease, the accumulation of the protein tau in the brain.

Previous studies had used mouse models of Alzheimer disease in which the protein amyloid- $\beta$ is overexpressed. Inclusions of amyloid- $\beta$ accumulate in the brains of individuals with Alzheimer disease and in the mouse model. Nicotine counteracted this accumulation in the mice.

In addition to amyloid- $\beta$, tau also accumulates in the brain of affected individuals. The researchers examined mice that overexpressed both amyloid- $\beta$ and tau, and exposed them to nicotine. Although the number of inclusions of amyloid- $\beta$ were not affected, the number of tau-containing tangles increased. The authors provide evidence that nicotine, through activation of acetylcholine receptors, may induce the upregulation of a protein that helps turn tau into tangles.

\section{Netting nonresponders}

A study in the January Public Library of Science Medicine hones methods to predict which patients will respond to drugs used to treat lung cancer.

Gefitinib (Iressa) and erlotinib (Tarceva) work most effectively for tumors with mutations in the gene encoding the drugs' target, the epidermal growth factor (EGF) receptor. But some tumors without the mutations respond, and there is a need for additional methods to predict which patients should receive treatment.

William Pao et al. $(1,7-61 ; 2005)$ provide evidence that tumors with mutations in the oncogene KRAS respond poorly to the drugs. The authors screened tumors from 60 individuals with lung cancer for mutations in $K R A S$, and treated them with gefitinib or erlotinib. The gene was normal in the 22 individuals who had responded to either drug, whereas the nine tumors that had abnormal KRAS genes did not respond.

Written by Charlotte Schubert

Mutations in the genes encoding KRAS and the EGF receptor are rarely found in the same tumor-suggesting that the proteins have similar roles in promoting lung cancer. Moreover, KRAS functions downstream of the EGF receptor, which might explain why tumors with KRAS mutations do not respond to inhibition of the EGF receptor. Larger studies should determine whether patients with KRAS mutations in their tumors should be spared treatment with the drugs.

\title{
Young blood
}

As humans and other animals age, their ability to regenerate damaged tissues from stem cells declines. A study in the 17 February Nature (433, 760-764) shows that blood from young mice puts spring back into aging stem cells. Irina Conboy et al. focused on the regeneration of muscle and liver, which have well-characterized stem cell populations. For instance, in aging muscle stem cells, signaling of the molecule Notch wanes. Previous work had suggested that stem cells in aging tissues had the capacity to divide and contribute to tissue regeneration-but that these cells were not receiving or interpreting the signals to do so. To ask whether stem cells in old and young animals were exposed to different signals, the researchers created mice that had fused circulatory systems. Old mice exposed to young blood were able to rebuild damaged muscle and liver. And, at the molecular level, their stem cells acted more like stem cells from younger animals: for instance, in muscle stem cells, Notch was activated and the cells divided. Moreover, stem cells in young mice slowed down when exposed to blood from old mice. The investigators next plan to uncover the responsible signals.

\section{Pollen power}

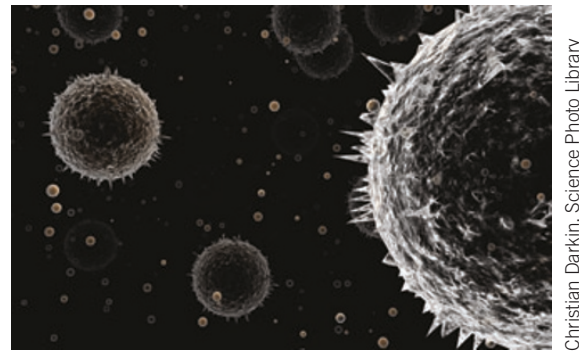

Pollen grains: oozing with fat

Pollen releases lipids that might compound the misery of hay fever, according to a study in the 21 February Journal of Experimental Medicine (201, 1-10). Experiments in cell culture suggest that such lipids determine the type of immune response that emerges in response to allergens.

Asthma is associated with a T helper type $2\left(\mathrm{~T}_{\mathrm{H}} 2\right)$ type of immune response, which leads mainly to the production of antibodies against allergens. Thelper type $1\left(\mathrm{~T}_{\mathrm{H}} 1\right)$ responses, in contrast, lead mainly to the production of immune cells that fight off invaders. Dendritic cells can direct whether $\mathrm{T}_{\mathrm{H}} 2$ or $\mathrm{T}_{\mathrm{H}} 1$ responses emerge in response to particular stimuli. But very little is known about how dendritic cells do this.

Heidrun Behrendt and colleagues had shown previously that lipids from pollen can cause the chemotaxis of certain immune cells in culture. The lipids, present on pollen along with allergens, resemble molecules that modulate the immune response, such as prostaglandins.

In the current study, the investigators show lipids from birch pollen nudge dendritic cells to direct a $\mathrm{T}_{\mathrm{H}} 2$ response. They incubated dendritic cells with a substance that activates them, such as bacterial lipopolysaccharide - and analyzed the effect of simultaneously added pollen lipids. One lipid, E-1 phytoprostane, had the most power. Dendritic cells incubated with this lipid induced naive $\mathrm{T}$ cells to develop typical $\mathrm{T}_{\mathrm{H}} 2$ responses, while those incubated without the lipid induced $\mathrm{T}_{\mathrm{H}} 1$ reponses. Whether this mechanism operates in vivo remains to be seen. 